\title{
454 - Quality of Life: People with Dementia and Their Caregiver in Indonesia
} Martina Wiwie Setiawan Nasrun ${ }^{1}$, Petrin Redayani ${ }^{1}$, Profitasari Kusumaningrum ${ }^{1}$, Hasya Layalia Lahino ${ }^{1}$ 1Department of Psychiatry, Faculty of Medicine, Universitas Indonesia

\begin{abstract}
Background: As a psychological being, quality of life is one of the most important part to human. Many things could affect one's quality of life, in elderly, Dementia is one of them. In 2013 approximately there were 960.000 people with dementia (PWD) in Indonesia. Caregiver who take care of PWDs' daily activity have an important role and maybe prone to stress, guilty feeling, anger and sad due to hardship of caregiving the PWD. Therefore caregivers' quality of life is substantial as PWDs' quality of life.
\end{abstract}

Aims: This study objective was to know the relationship of quality of life people with dementia and their caregiver in Indonesia.

Methods: A descriptive-analytic study was conducted in RSUPN Dr. Ciptomangunkusumo Hospital Jakarta. Eighty four subjects were participated (42 PWD and 42 caregivers). PWD subjects were interviewed using questionnaires EQ-5D and severity of dementia measured using MMSE. Meanwhile caregivers' QOL was measured using WHOQOL-BREF. Data was analyzed using binary correlation between PWD and Caregivers' quality of life.

Results: There was no correlation of PWD physical health and caregivers' quality of life. However there was strong correlation between quality of health PWD and caregivers'QOL notably in environmental domain $(r=0.839)$. And there were also a strong correlation between PWD severity of dementia and caregiver's QOL inphysical domain $(r=0.946)$. Some of caregivers' factor influencing quality of life were age $(r=0.805)$, duration of caregiving each day $(r=0.362)$ and experience of caregiving $(r=0.927)$

Conclusion: Study found that the quality of health PWD had a strong correlation with caregiver's QOL specifically in environmental domain.Internal factors related to caregiversQOL were age, duration of caregiving each day and experience of caregiving. Future study should be focused on objective measurement of quantify health quality.These findings suggest that caregivers' quality of life also an essential part in managing dementia.

Keyword: person with dementia (PWD), caregiver, quality of life (QOL) 